\title{
Resveratrol alleviates sepsis-induced acute kidney injury by deactivating the IncRNA MALAT1/MiR-205 axis
}

\author{
BIAO WANG ${ }^{1}$, YIJUN WANG ${ }^{2}, K E X^{2}$, ZHENHUA ZENG ${ }^{3}$, ZIQIANG XU ${ }^{2}$, DONGYOU YUE ${ }^{2}$, \\ TAO LI ${ }^{2}$, JIHUI LUO', JUNHONG LIU' ${ }^{2}$ JIEMIN YUAN ${ }^{2}$
}

${ }^{1}$ The Second Hospital, University of South China, China

${ }^{2}$ Chenzhou No. 1 People's Hospital, China

${ }^{3}$ Nanfang Hospital, Southern Medical University, China

\begin{abstract}
Introduction: Resveratrol plays a protective role against sepsis development, and the long noncoding RNA (IncRNA) MALAT1 is an inflammation-relevant biomarker. This investigation attempted to reveal whether resveratrol attenuated inflammation of sepsis-induced acute kidney injury (AKI) by regulating MALATI.

Material and methods: In total 120 rats were divided into a control group $(n=20)$, a Sham group $(n=20)$, a sepsis group $(n=40)$ and a resveratrol group $(n=40)$, and serum levels of inflammatory cytokines and AKI biomarkers were determined. An equal number of rats under identical treatments were, additionally, tracked for their survival, and the serum level of IncRNA MALATI was measured by RT-PCR. Moreover, septic cell models were constructed by treating HK-2 cells with lipopolysaccharide $(L P S)$, and tumor necrosis factor $\alpha(T N F-\alpha)$, interleukin $(I L)-1 \beta$, IL-6 levels released by the cells were determined with ELISA.

Results: Resveratrol treatment significantly brought down serum levels of inflammatory cytokines (i.e. $T N F-\alpha, I L-1 \beta$ and IL-6), kidney function indicators (i.e. Scr, blood urea nitrogen [BUN] and Scys $C$ ), AKI biomarkers (i.e. NGAL and KIM-1) and MALATI in cecal ligation and puncture (CLP)-induced septic model rats (all $p<0.05)$, and the life span of septic rats was elongated by resveratrol treatment $(p<0.05)$. Viability and cytokine release of LPS-treated HK2 cells were rescued by resveratrol $(p<0.05)$, which was accompanied by a marked fall of MALATI expression $(p<0.05)$. In addition, siMALAT1 diminished viability and suppressed cytokine release of HK2 cells, while pcDNA3.1-MALAT1 hindered the impact of resveratrol on the inflammatory response of HK2 cells $(p<0.05)$. Ultimately, miR-205, a protective molecule in sepsis-relevant AKI, was down-regulated by resveratrol and si$\operatorname{MALAT1}(p<0.05)$.

Conclusions: Resveratrol relieved sepsis-induced AKI by restraining the IncRNA MALATI/miR205 axis.
\end{abstract}

Key words: sepsis, acute kidney injury, inflammation, resveratrol, IncRNA MALAT1, miRNA-205, cell viability.

\section{Introduction}

Sepsis, a systemic inflammatory response syndrome (SIRS) stimulated by infection, is usually complicated by acute kidney injury (AKI) [1-3], which contributes to a high mortality of 40-60\% [4]. Lipopolysaccharide (LPS), an ingredient of gram-negative bacteria, has been commonly applied to establish cell models of sepsis-induced AKI [5]. It was documented that LPS-treated renal tubular epithelial cells could overproduce inflammatory cytokines, such as interleukin 
named Takaoka. This natural antibiotic not merely fought against bacteria-/fungus-induced infections, but also prevented onset of cancers, cardiovascular diseases and inflammatory disorders [9-11]. Of note, it was reported that resveratrol attenuated inflammation in AKI by activation of nuclear factor-erythroid-2-related factor 2 (Nrf2) signaling [12] and suppression of nuclear factor- $\kappa \mathrm{B}(\mathrm{NF}-\kappa \mathrm{B})$ signaling [13], which implied that resveratrol was likely to be involved in the etiology of sepsis-relevant AKI by regulating molecular networks.

A myriad of evidence has demonstrated that resveratrol modulates disease progression by altering expression of miRNAs [14-16]. For instance, levels of miR-663, miR-146a and miR-21, crucial biomarkers of sepsis onset $[1,17,18]$, were up-regulated in the resveratrol-treated SW480 cell line [19]. Notably, lncRNAs were documented to sponge miRNAs, so as to reduce inhibition of miRNAs on disease-related genes. Taking sepsis for example, lncRNA SNHG16 was found to up-regulate TLR4 expression in LPS-treated RAW264.7 cells by binding to miR-15a/16 [20]. However, IncRNAs that were involved in resveratrol's ameliorating sepsis-induced AKI have rarely been investigated.

LncRNA MALAT1, initially speculated as a tumor biomarker [21], revealed positive correlations with serum levels of TNF- $\alpha$, IL-1 $\beta$, IL-6, IL-10, IL-17 and interferon $\gamma$ (IFN- $\gamma$ ) in septic rats [22], and an incremental level of MALAT1 was suggestive of unfavorable survival among sepsis patients [23]. MALAT1 was also reported to exacerbate sepsis-triggered cardiac inflammation by interacting with miR-125b [22]; nonetheless, little was known about the significance of MALAT1 in sepsis-derived AKI. Interestingly, several MALAT1-sponged miRNAs were documented to participate in AKI etiology; for example, miR204, down-regulated by MALAT1 in lung adenocarcinoma [24], was able to relieve sepsis-caused AKI [25]. Moreover, miR-205, sponged by MALAT1 in Parkinson's disease (PD) [26], reduced the likelihood of AKI onset among sepsis patients, and patients carrying lowly-expressed miR205 tended to enjoy a longer life span than those with high miR-205 expression [27]. Although MALAT1 might be involved in sepsis-derived AKI by regulation of miRNAs, whether it was implicated in the protective effect of resveratrol against sepsis-caused AKI remained uncertain.

Therefore, this study was intended to determine whether resveratrol attenuated sepsis-triggered AKI by down-regulating MALAT1, which might provide new treatment strategies for sepsis patients.

\section{Material and methods}

\section{Establishment of sepsis models in rats}

\section{Detection of cytokines in serum of rats}

120 male specific-pathogen-free male Sprague-Dawley (SD) rats, aged 13-15 weeks old and weighing 200-250 g, were provided by the experimental animal center of No. 1 People's Hospital (First Affiliated Hospital of Xiang Nan University) (Hu'nan province, China). These rats were randomly divided into a blank control group $(n=20)$, a Sham operation group (Sham group, $n=20$ ), a cecal ligation and puncture (CLP) group (Sep group, $n=40$ ) and a resveratrol group (Res group, $n=40$ ), and they were fasted for $12 \mathrm{~h}$ prior to surgery, with free access to water. The abdomen of rats in the Sham group was closed immediately after laparotomy, while the ceca of rats in the CLP group and Res group were ligated and punctured before abdominal closure. In the wake of successful modeling, rats of the Res group were injected with $0.3 \mathrm{ml} / 100 \mathrm{~g}$ Res solution (Catalog number: $13065-54-8$, purity $\geq 99 \%$, Hubei shengtian henchuang pharmaceutical corporation, China) from the tail vein, and rats of the other groups were injected with equal amounts of normal saline. Around $12 \mathrm{~h}$ after surgery, $5 \mathrm{ml}$ of blood was extracted from the abdominal aorta of each rat, and the blood samples were centrifuged at $5000 \mathrm{r} / \mathrm{min}$ for $15 \mathrm{~min}$ to determine serum levels of cytokines and MALAT1: 1) serum levels of NGAL, KIM-1, TNF- $\alpha$, IL- $1 \beta$ and IL- 6 were determined with corresponding ELISA kits (BD, USA), 2) serum levels of Scr, blood urea nitrogen (BUN) and Scys C were measured using a MedLab automated biomedical analyzer (Nanjing medease science and technology corporation, China), and 3) serum level of MALAT1 was detected via PCR as detailed below.

\section{Tracking survival of model rats}

Another population of rats $(n=120)$ under the same treatments, including a control group $(n=20)$, a Sham group $(n=20)$, a Sep group $(n=40)$ and a Res group $(n=40)$, was tracked for survival, and blood samples were taken immediately after death of the rats or until end of the observation period.

All animal experiments in this study were conducted according to the requirements of Care and Use of Experimental Animals of the US National Institutes of Health (Bethesda, MD, USA), and this program has obtained approval from the Animal Care Committee of No. 1 People's Hospital (First Affiliated Hospital of Xiang Nan University) (Hu'nan province, China).

\section{Cell culture}

Human kidney proximal tubular epithelial cell 2 (HK2) cell line, purchased from American Type Culture Collection (ATCC, USA), was inoculated in minimum Eagle's medium (MEM), which contained 10\% fetal bovine serum (FBS), $1 \%$ penicillin-streptomycin and $1 \%$ non-essential amino acids (NEAA), under an atmosphere of $37^{\circ} \mathrm{C}$ and $5 \% \mathrm{CO}_{2}$.

\section{Cell transfection}

HK2 cells seeded within 6-well plates were cultured for $24 \mathrm{~h}$, until cell confluence occurred at $90 \%$. Then siMALAT1, si-NC, pcDNA3.1 and pcDNA3.1-MALAT1, 
all purchased from Ribobio (China), were separately transfected into HK2 cells for $48 \mathrm{~h}$, as per guidance of the Lipofectamine 2000 transfection kit (Invitrogen, USA).

\section{Cell treatment with LPS and resveratrol}

HK2 cells were treated through 3 approaches: 1) LPS treatment: addition of $10 \mu \mathrm{g} / \mathrm{ml} \mathrm{LPS}$ (Sigma, USA), 2) PBS treatment: addition of equal volumes of PBS, and 3) Res + LPS treatment: supplementation of $200 \mu \mathrm{mol} / 1$ resveratrol (Enzo life sciences, USA) for $2 \mathrm{~h}$ and then addition of $10 \mu \mathrm{g} / \mathrm{ml}$ LPS. After 6-h cell culture, supernatant of HK2 cells was collected after centrifugation, and then they were stored at $-80^{\circ} \mathrm{C}$ in the refrigerator.

\section{Real-time polymerase chain reaction (PCR)}

After extraction from blood samples and HK2 cells with the TRIzol method (TaKaRa, Japan), density and purity of total RNAs were measured by an ultraviolet spectrometer (HITACHI, Japan). Then cDNAs of MALAT1 were transformed from RNAs according to the manual of the PrimeScript RT reagent kit (TaKaRa, Japan), and cDNAs of miR205 were synthesized in the light of specifications of the One Step miRNA PrimeScript cDNA synthesis kit (TaKaRa, Japan). Using primers of target genes (Table 1), the cDNAs were amplified in line with instructions of the SYBR Premix Ex Taq II kit (TaKaRa, Japan). Relative expression levels of genes were determined using the $2^{-\Delta \Delta \mathrm{Ct}}$ method, and gene levels in the control group were normalized to 1 .

\section{CCK8 assay to assess proliferation of $\mathrm{HK} 2$ cells}

HK2 cells of the logarithmic growth phase were inoculated into 96-well plates at the density of $5 \times 10^{4} / \mathrm{ml}$ for $48 \mathrm{~h}$. Subsequently, HK2 cells of each well were treated with $10 \mu \mathrm{l}$ of CCK8 solution (Beyotime, USA) for $4 \mathrm{~h}$, and the absorbance value $(\mathrm{A})$ of cell samples was measured at the wavelength of $570 \mathrm{~nm}$ using a microplate reader (model: BIORAD-550, Bio-Rad, USA).

\section{Determination of cytokines secreted by HK2 cells}

Levels of IL-1 $\beta$, IL- 6 and TNF- $\alpha$ in the supernatant of HK2 cells were detected with corresponding ELISA kits (BD, USA).

\section{Statistical analyses}

All the data were statistically analyzed by means of SPSS13.0 statistical software. Quantitative data, all presented as mean \pm standard deviation (SD), were compared with Student's $t$ test or one-way analysis of variance (ANOVA), and comparison of enumeration data was conducted with the $\chi^{2}$ test. Pearson correlation was implemented to analyze correlations between serum MALAT1 level and biomarker levels, and Kaplan-Meier curves were plotted to estimate the association of serum MALAT1 level with survival of rats. $P$ value $<0.05$ was deemed statistically significant.

\section{Results}

Resveratrol affected serum levels of MALAT1, as well as indicators of inflammatory response, kidney function and $A K I$, in the septic rat model

Rats of the Sep group had significantly higher serum levels of TNF- $\alpha$, IL- $1 \beta$ and IL- 6 than rats of the Sham group (all $p<0.001$ ), and levels of the cytokines were significantly lower in the Sep + Res group as compared with the Sep group (all $p<0.001$ ) (Fig. 1A). In addition, Scr, BUN and Scys C levels, suggestive of renal function [29], were markedly raised in septic model rats as compared to rats of the Sham group (all $p<0.001$ ), yet administration of resveratrol (i.e. Rep group) significantly reduced Scr, BUN and Scys C levels in comparison to the Sep group (all $p<0.001$ ) (Fig. 1B). When compared with rats of the Sham group, NGAL and KIM-1 levels, pivotal indicators of AKI [30], were increased in septic model rats (all $p<0.001$ ),

Table 1. Primers for genes

\begin{tabular}{lcc}
\hline Genes & Forward $\left(\mathbf{5}^{\prime} \mathbf{-} \mathbf{3}^{\prime}\right)$ & Reverse $\left(\mathbf{5}^{\prime} \mathbf{-} \mathbf{3}^{\prime}\right)$ \\
\hline Human (HK2 cell line) & AAAGTCCGCCATTTTGCCAC & \\
\hline MALAT1 & ACCCACTCCTCCACCTTTGAC & ACAACTCGCATCACCGGAAT \\
\hline GAPDH & GCGGCGGTCCTTCATTCCACCG & TGTTGCTGTAGCCAAATTCGTT \\
\hline miR-205 & GCTTCGGCAGCACATATACT & ATCCAGTGCAGGGTCCGAGG \\
\hline U6 & & ACGCTTCACGAATTTGCGTG \\
\hline Model rats & CTATGCTGTTGGCACGACA & \\
\hline MALAT1 & CAATGACCCCTTCATTGACC & TCCTGAGGTGACTGTGAACC \\
\hline GAPDH & GCGGCGGTCCTTCATTCCACCG & GACAAGCTTCCCGTTCTCAG \\
\hline miR-205 & TTCGTGAAGCGTTCCATATTTT & ATCCAGTGCAGGGTCCGAGG \\
\hline U6 & & ACGCTTCACGAATTTGCGTG \\
\hline
\end{tabular}


A
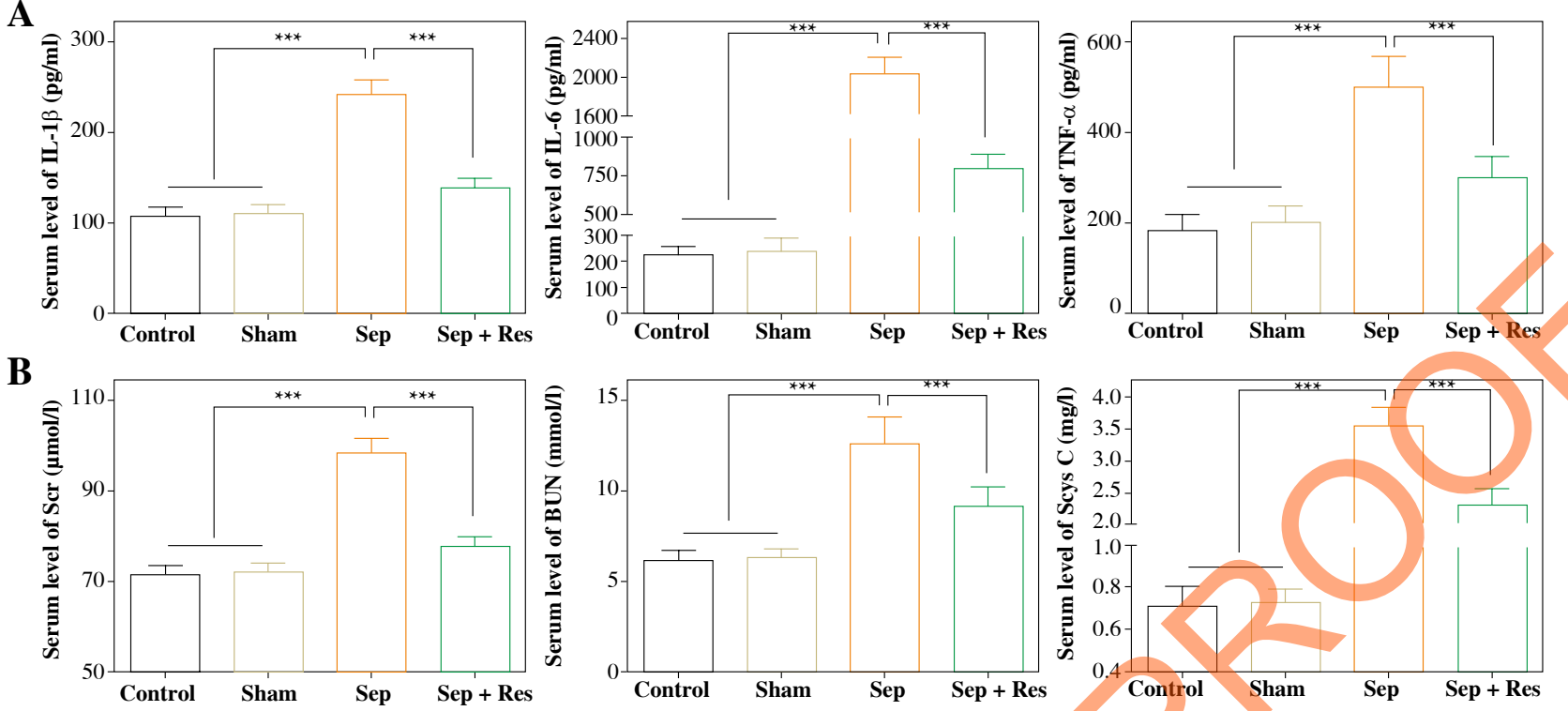

C
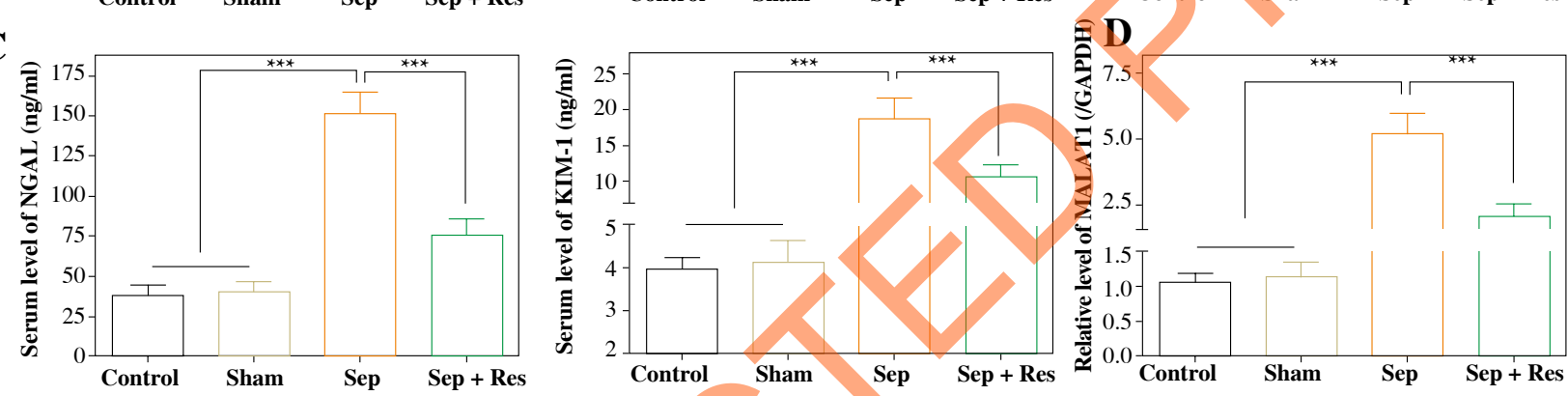

Fig. 1. Indicators of inflammation response $(\mathbf{A})$, renal function $(\mathbf{B})$ and acute kidney injury $(\mathbf{C})$, as well as serum level of lncRNA MALAT1 (D), were compared among model rats of control group, Sham group, Sep group and Sep + Res group. $* * * p<0.001$

while resveratrol treatment (i.e. Rep group) mitigated AKI injuries in septic rats (all $p<0.001$ ) (Fig. 1C). Moreover, serum level of MALAT1 was raised in model rats of Sep group (all $p<0.001$ ), but it fell markedly in the Sep + Res group as compared with Sep group (all $p<0.001)$ (Fig. 1D).

\section{MALAT1 level was correlated with biomarker levels indicative of inflammation, kidney function and AKI in serum of resveratrol-treated septic model rats}

MALAT1 level was positively correlated with serum levels of IL-1 $\beta$ (Fig. 2A), IL-6 (Fig. 2B) and TNF- $\alpha$ (Fig. 2C) in model rats of sepsis group (IL-1 $\beta$ : $r_{\mathrm{s}}=0.358, p<0.001$; IL-6: $r$ s $=0.481, p<0.001$; TNF- $\alpha: r^{2}=0.349, p<0.001$ ) and Sep + Res group (IL-1 $\beta: r^{2}=0.576, p<0.001$; IL-6: $r^{2}=0.325, p<0.001$; TNF- $\alpha: r^{2}=0.596, p<0.001$ ). There were also pronounced correlations between MALAT1 level and levels of Scr (Fig. 3A), BUN (Fig. 3B) and Scys C (Fig. 3C) among rats of sepsis group (Scr: $r^{2}=0.497, p<0.001$; BUN: $r^{2}=0.348, p<0.001$; Scys C: $r^{2}=0.228, p=0.002$ ) and rats of Sep + Res group (Scr: $r^{2}=0.548, p<0.001$;
BUN: $r^{2}=0.615, p<0.001$; Scys C: $r^{2}=0.401, p<0.001$ ). Furthermore, MALAT1 level rose significantly as NGAL (Fig. 4A) and KIM-1 (Fig. 4B) levels were elevated in model rats of the sepsis group (NGAL: $r_{\mathrm{s}}=0.421, p<0.001$; KIM-1: $\left.r_{\mathrm{s}}=0.484, p<0.001\right)$ and Sep + Res group (NGAL: $\left.r_{\mathrm{s}}=0.613, p<0.001 ; \mathrm{KIM}-1: r_{\mathrm{s}}=0.577, p<0.001\right)$. Nonetheless, hardly any significance was discerned in the correlation between serum level of MALAT1 and serum level of IL-1 $\beta(p=0.302)$, IL-6 $(p=0.827)$, TNF- $\alpha(p=0.128)$, Scr $(p=0.881)$, BUN $(p=0.711)$, Scys C $(p=0.758)$, NGAL $(p=0.492)$ or KIM-1 $(p=0.402)$ (Figs. $2-4)$.

\section{MALAT1 level was predictive of survival among septic model rats}

Among 120 model rats whose survival status was tracked, rats of the Sep group demonstrated a higher MALAT1 level than rats of the Sham group $(p<0.001)$, while rats of the Rep group had a much lower average MALAT1 level than rats of the Sep group $(p<0.001)$ (Fig. 5A). Furthermore, survival of rats in the sepsis group was quite unfavorable as compared with rats of the Sham group and control group $(p<0.001)$, and septic rats under 
A

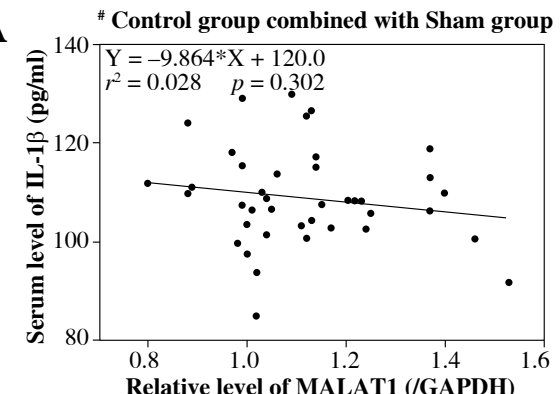

B

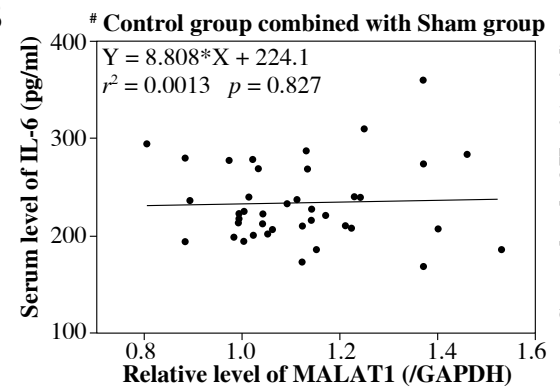

C

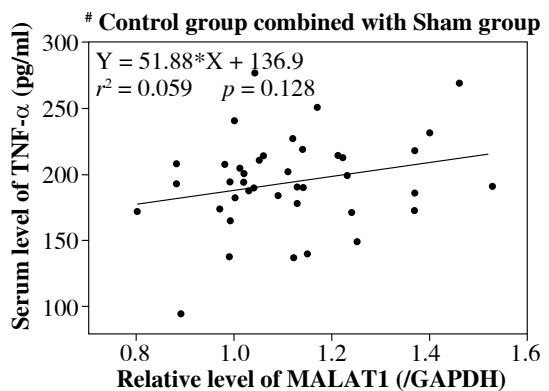

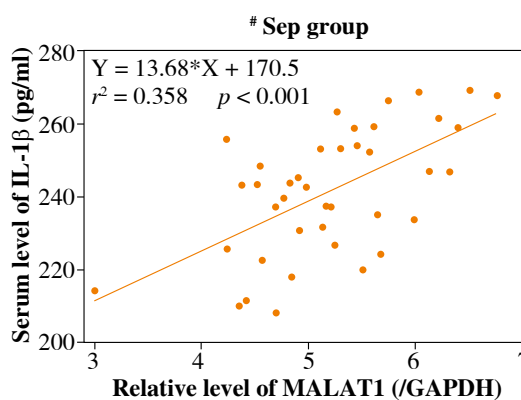
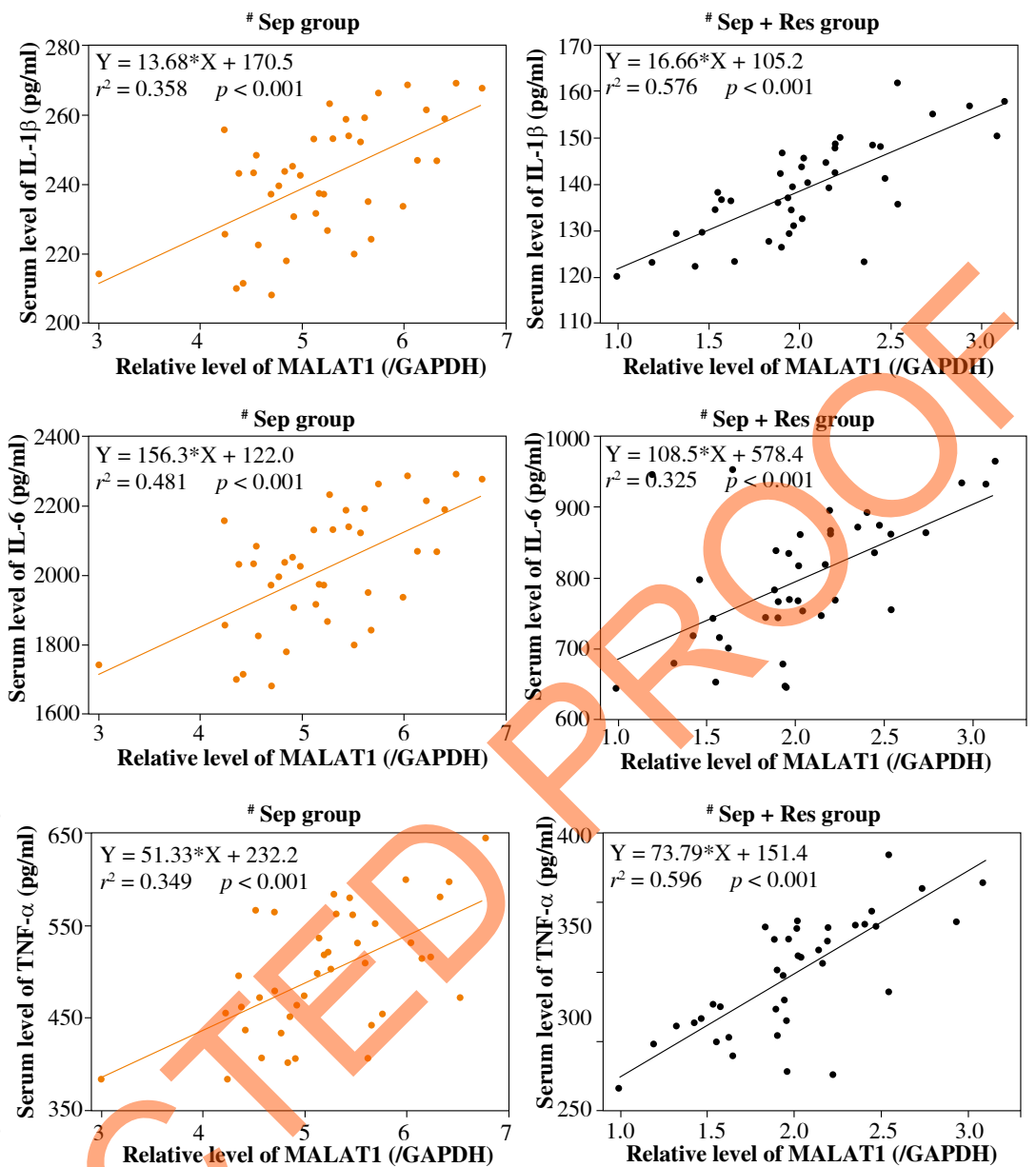

Fig. 2. LncRNA MALAT1 level was correlated with serum levels of IL-1 $\beta$ (A), IL-6 (B) and TNF- $\alpha$ (C) among rats of control group combined with Sham group combined, Sep group and Sep + Res group

resveratrol treatment (i.e. Sep + Res group) demonstrated longer survival than septic model rats $(p<0.05)$ (Fig. 5B). With median MALAT1 level (i.e. 7.07) as the cut-off point, we found that septic rats with a high ( $>$ median) MALAT1 level revealed shorter survival than rats with a low $(\leq$ median) MALAT1 level $(p<0.01)$ (Fig. 5C). Also using median MALAT1 level as the dividing point, rats of the Sep + Res group enjoyed more ideal survival if they had a low $(\leq 2.56)$ MALAT1 level, with the high-level (> 2.56) MALAT1 group as the reference $(p<0.001)$ (Fig. 5D).

\section{Resveratrol diminished viability and cytokine release of $\mathrm{HK} 2$ cells by down-regulating} MALAT1 expression

MALAT1 expression increased markedly in LPS-treated HK2 cells $(p<0.001)$, yet resveratrol treatment (i.e. LPS + resveratrol group) gave rise to a sharp decrease of MALAT1 expression in septic cell models $(p<0.001)$ (Fig. 6A). Moreover, MALAT1 expression was up-regulated in HK2 cells transfected by pcDNA3.1- MALAT1 $(p<0.001)$, yet was down-regulated as MALAT1 was silenced $(p<0.001)$ (Fig. 6B).

Viability of HK2 cells was strengthened after stimulation of LPS, when compared with the control group $(p<0.001)$ (Fig. 6C). Both resveratrol and si-MALAT1 were capable of undermining viability of LPS-treated HK2 cells $(p<0.001)$, although resveratrol played a stronger restraining role than siMALAT1 $(p<0.001)$. Also the suppressive effect of resveratrol (i.e. LPS + resveratrol group) on viability of LPS-treated HK2 cells could be alleviated by pcDNA3.1-MALAT1 (i.e. LPS + resveratrol + pcDNA3.1-MALAT1 group) $(p<0.001)$.

In addition, TNF- $\alpha$, IL- $1 \beta$ and IL- 6 levels were dramatically up-regulated in supernatants of HK2 cells after induction of LPS $(p<0.001)$ (Fig. 6D-F). Nevertheless, the cytokine levels were decreased in HK2 cells of the LPS + resveratrol group and LPS + si-MALAT1 group relative to HK2 cells of the LPS group $(p<0.001)$. Moreover, TNF- $\alpha$, IL- $1 \beta$ and IL-6 levels in the LPS + resveratrol group were up-regulated by extra transfection of pcDNA3.1-MALAT1 (i.e. resveratrol + LPS + pcDNA3.1-MALAT1 group) $(p<0.001)$. 
A

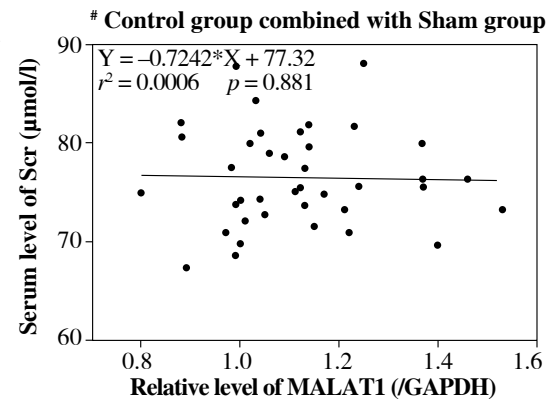

B

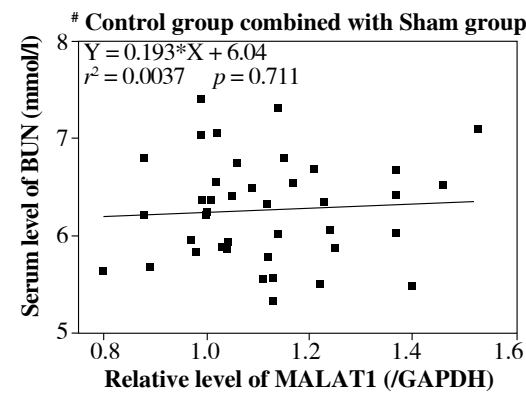

C

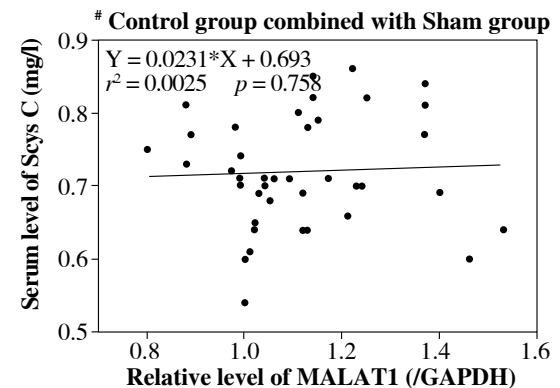

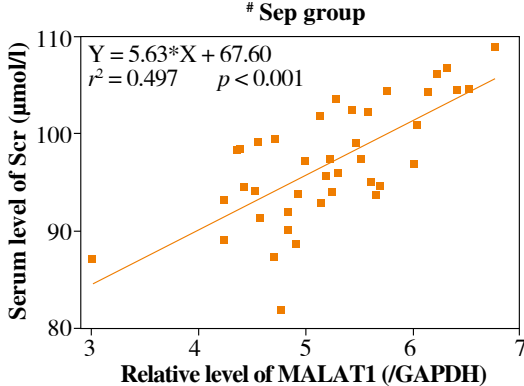
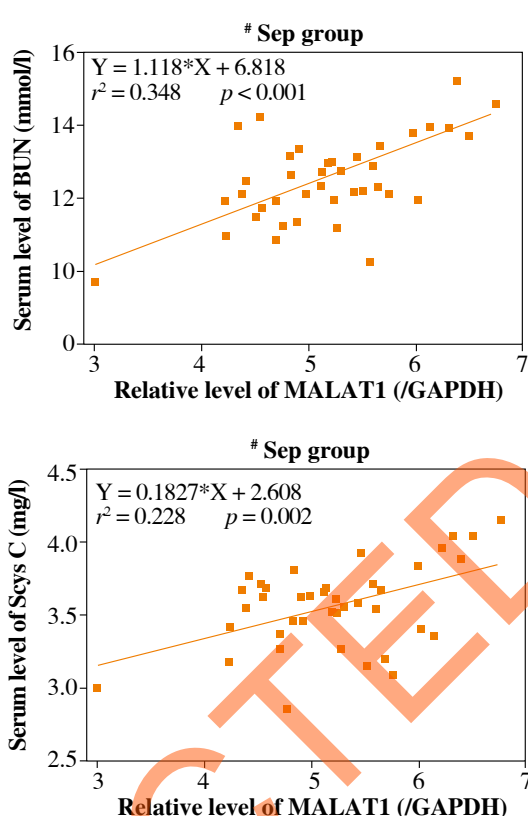
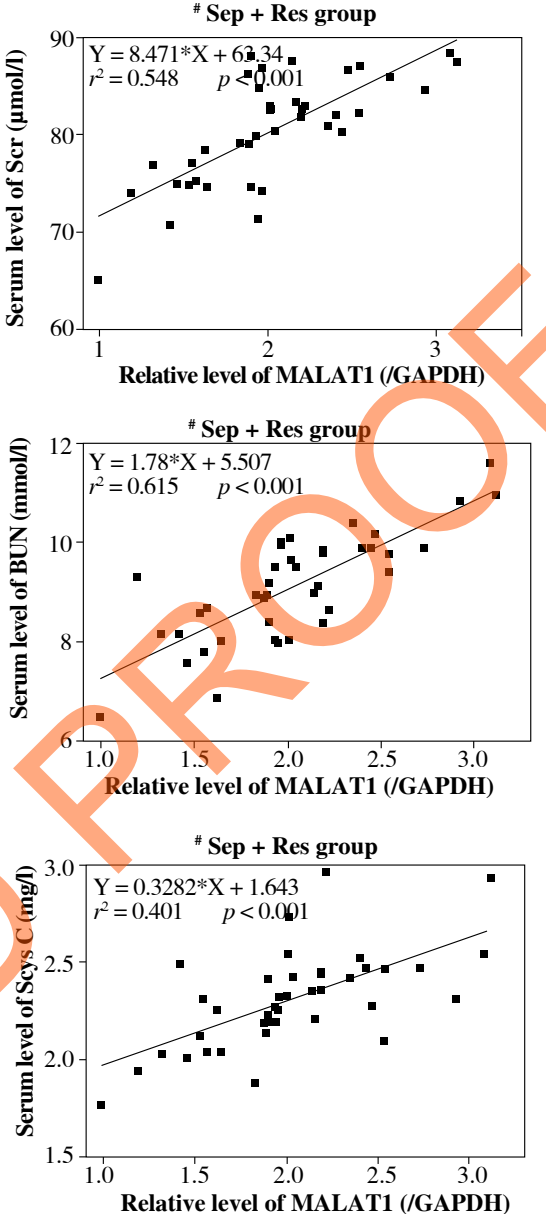

Fig. 3. LncRNA MALAT1 level exhibited relevance to serum levels of creatine (Scr) (A), BUN (B) and Scys C (C) among rats of control group combined with Sham group, Sep group and Sep + Res group

\section{Resveratrol and MALAT1 impacted miR-205 expression in $\mathrm{HK} 2$ cells}

MiR-205 expression in HK2 cells was raised after transfection of si-MALAT1 $(p<0.001)$, and was reduced by pcDNA3.1-MALAT1 $(p<0.001)$ (Fig. 6G). Moreover, miR-205 expression exhibited a drop after LPS treatment $(p<0.001)$, while resveratrol (i.e. resveratrol + LPS group) and si-MALAT1 (i.e. si-MALAT1 + LPS group) both up-regulated miR-205 expression in LPS-treated HK2 cells $(p<0.001)$ (Fig. 6H). Moreover, transfection of pcDNA3.1-MALAT1 (i.e. resveratrol + LPS + pcDNA3.1-MALAT1 group) led to increased miR-205 expression in resveratrol + LPS-treated HK2 cells $(p<0.001)$ (Fig. 6H).

\section{Discussion}

Up to $19 \%$ of sepsis patients simultaneously develop AKI [31], and a large number of them often miss the golden timing for treatment [6], owing to the absence of sensitive diagnostic biomarkers [32]. Therefore, to benefit patients with sepsis-induced AKI, investigating its underlying etiology is necessary.

It was noteworthy that the inflammatory cascade was intensified during septic progression, which could severely damage renal function [33]. In this investigation, we also discovered that inflammation was abnormally magnified, along with destroyed renal function, in CLP-induced septic model rats; however, this physiological change was relieved after resveratrol injection (Fig. 1). Multifold signaling pathways have been documented to explain the protective role of resveratrol against inflammation-caused damage [34]. For instance, TLR2/NF- $\mathrm{KB}$ signaling and mitogen-activated protein kinase (MAPK) signaling, both motivated by LPS, could be deactivated by resveratrol treatment, so that exacerbation of inflammatory diseases was prevented [35, 36]. Expanding perceptions of this aspect, this investigation newly revealed that lncRNA MALAT1 which was up-regulated in CLP-induced septic model rats was, to some extent, normalized after resveratrol treatment (Fig. 1D). Furthermore, given that variation of MALAT1 expression was reflective 

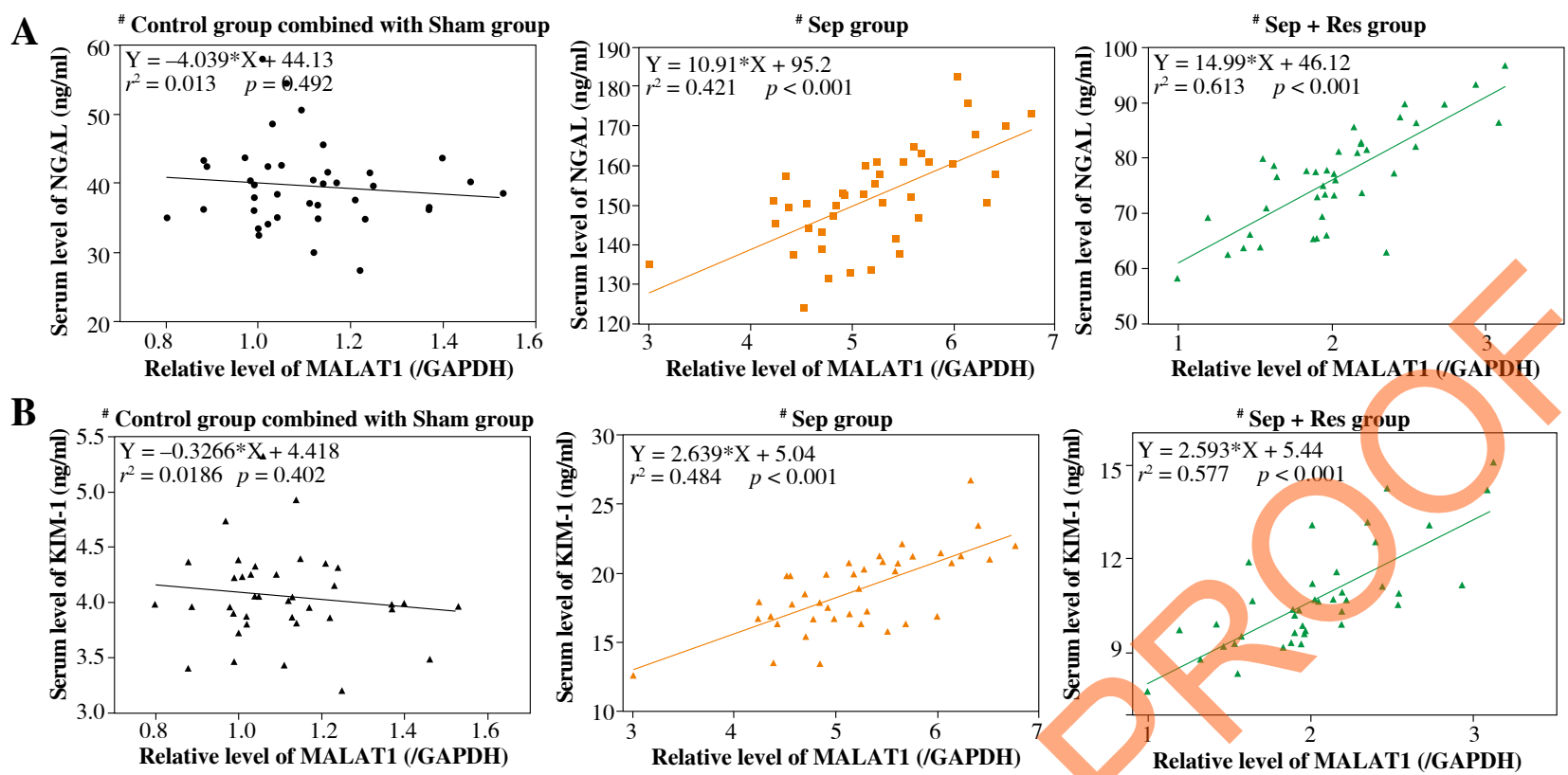

Fig. 4. LncRNA MALAT1 level demonstrated associations with serum levels of NGAL (A) and KIM-1 (B) among rats of control group combined with Sham group, Sep group and Sep + Res group
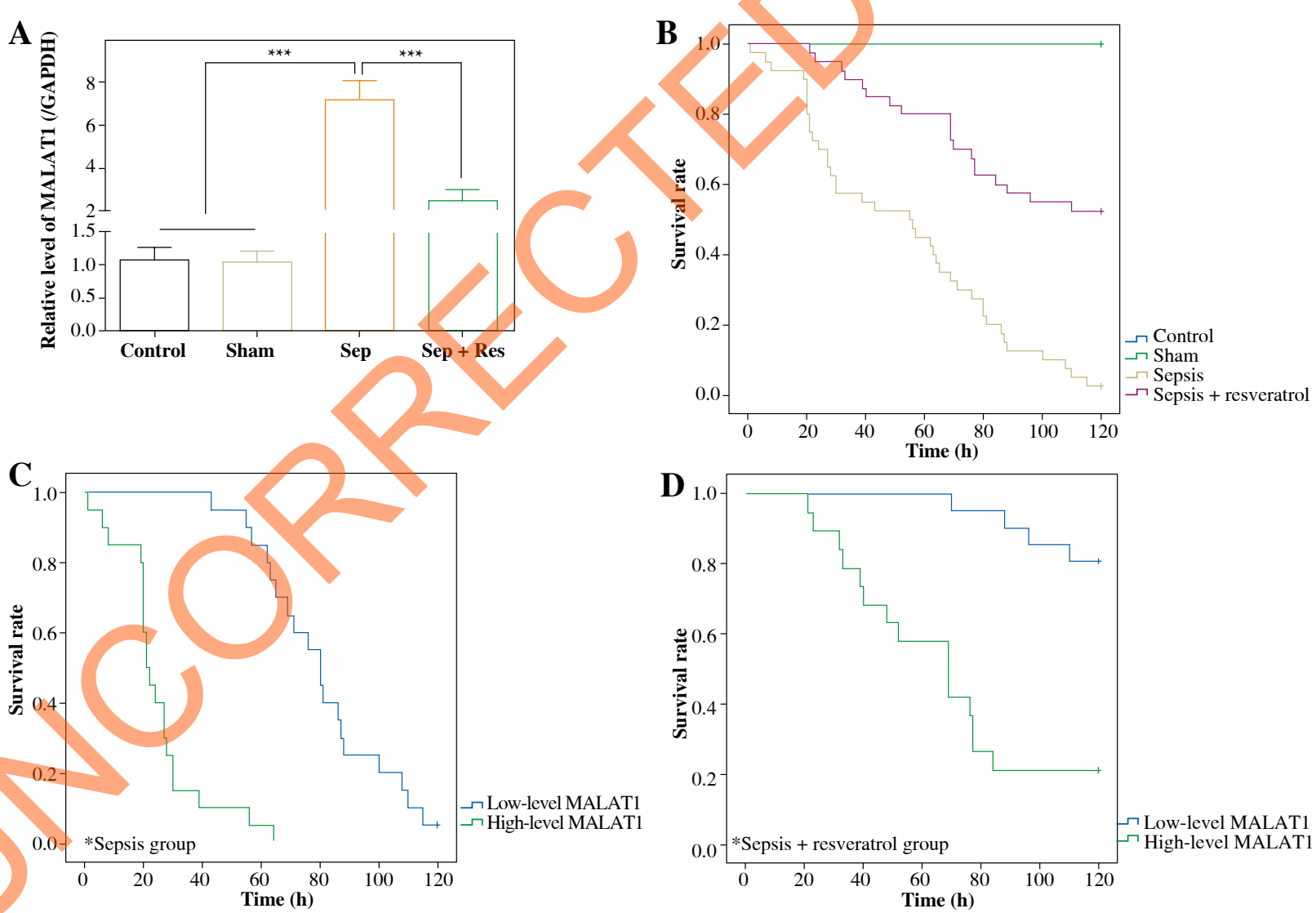

Fig. 5. Association of IncRNA MALAT1 level with survival of model rats under different treatments. A) Serum level of lncRNA MALAT1 was determined among model rats of control group, Sham group, Sep group and Sep + Res group. *** $p<0.001$. B) Survival rates of model rats were compared among control group, Sham group, Sep group and Sep + Res group. C) Survival rates of model rats in the sepsis group were compared between high-level MALAT1 group and low-level MALAT1 group. D) Survival of model rats in the Sep + Res group was compared between high-level MALAT1 group and low-level MALAT1 group 

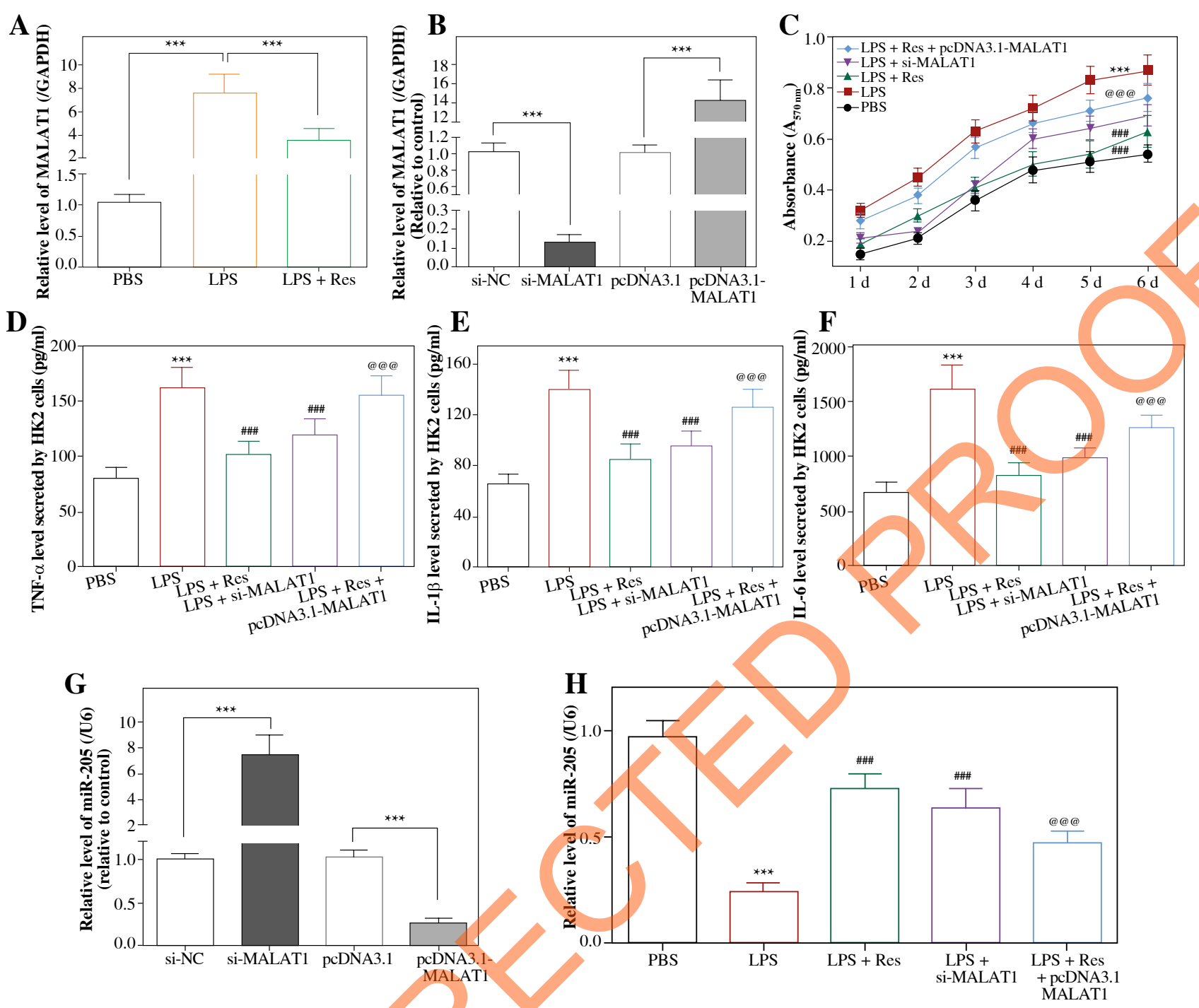

Fig. 6. Resveratrol reduced proliferation and inflammatory response of HK2 cells by regulating lncRNA MALAT1 and miR-205. A) MALAT1 expression was determined in HK2 cells of PBS group, LPS group and LPS + Res group. $* * * p<0.001$. B) MALAT1 expression in HK2 cells was altered after transfection of si-MALAT1 and pcDNA3.1MALAT1. $* * * p<0.001$. C) Viability of HK2 cell line was determined after treatments of PBS, LPS, LPS + Res, LPS + si-MALAT1 and LPS + Res + pcDNA3.1-MALAT1. ${ }^{* * *} p<0.001$ in comparison to PBS group; ${ }^{\# \#} p<0.001$ in comparison to LPS group; ${ }^{@ @} @ p<0.001$ in comparison to LPS + Res group. D-F) TNF- $\alpha$ (D), IL-1 $\beta$ (E) and IL-6 (F) levels were compared among PBS group, LPS group, LPS + Res group, LPS + si-MALAT1 group and LPS + Res + pcDNA3.1-MALAT1 group. ${ }^{* * *} p<0.001$ in comparison to PBS group; ${ }^{\# \#} p<0.001$ in comparison to LPS group; @@@ $p<0.001$ in comparison to LPS + Res group. G) MiR-205 expression in HK2 cells was measured after transfection of si-MALAT1 and pcDNA3.1-MALAT1. ***p $<0.001$. H) MiR-205 expression in HK2 cells was compared among PBS group, LPS group, LPS + Res group, LPS + si-MALAT1 group and LPS + Res + pcDNA3.1-MALAT1 group. $* * * p<0.001$ in comparison to PBS group; ${ }^{* \#} p<0.001$ in comparison to LPS group; ${ }^{@ @ @ ~} p<0.001$ in comparison to LPS + Res group

of renal function (Figs. 3-4), inflammatory response (Fig. 2) and survival status (Fig. 5) of septic model rats, resveratrol was likely to ameliorate sepsis-relevant AKI by inhibiting MALAT1-led signaling pathways.

LncRNAs have displayed huge potential with respect to averting inflammatory injuries through regulat- ing mitochondrial division, pro-inflammatory cytokines, calcium ion transport and NF- $\kappa \mathrm{B}$ signaling [37, 38]. For example, linc 0206, linc 3995, linc7190 and linc 7705 powerfully down-regulated TNF- $\alpha$ and IL-6 expression in Pam3CSK4-stimulated THP1 macrophages [39], while expressions of E-selectin, vascular cell adhesion 
molecule-1 (VCAM-1), IL-8 and IL-6 were negatively modulated by lncRNA human interleukin 7 receptor (lncIL7R) in LPS-treated THP1 macrophages [40]. Concerning MALAT1 studied here, which was over-expressed in LPS-treated macrophages [41], its low expression could protect myocardia from inflammatory damage by sponging miR-26a and down-regulating TLR4 [42], and ERK/ MAPK signaling, pivotal to AKI development [43], might be activated by MALAT1 [44]. These proofs all supported the role of MALAT1-led signaling pathways in protecting against sepsis-relevant inflammation and AKI, providing a molecular approach that resveratrol depended on to fight against sepsis-induced AKI.

Additionally, cell models were built to confirm the association of the resveratrol/MALAT1 axis with sepsis-relevant inflammation (Fig. 6). We observed that HK-2 cells treated with LPS released higher levels of IL-6, IL-1 $\beta$ and TNF- $\alpha$ than the control group (Fig. 6D-F), indicating that sepsis-induced AKI cell models were successfully established $[5,45]$. Furthermore, resveratrol was discovered to repress viability (Fig. 6C) and decrease IL-6, IL-1 $\beta$ and TNF- $\alpha$ production in LPS-treated HK2 cells (Fig. 6D-F), implying in vitro protection of resveratrol against inflammation injury. More interestingly, MALAT1 level varied when HK2 cells was managed by resveratrol (Fig. 6A), and si-MALAT1, similar to resveratrol, also protected HK2 cells from being destroyed by LPS (Fig. 6D-F). Additionally, pcDNA3.1-MALAT1 could, to some extent, prohibit the effect of resveratrol on viability and cytokine production of HK2 cells (Fig. 6C-F), which all suggested that MALAT1 was subjected to in-vitro regulation of resveratrol. Furthermore, miR-205 expression, previously reported to prevent sepsis-triggered AKI [27], was raised by resveratrol and si-MALAT1 (Fig. 7G, H), providing a hint that the MALAT1/miR-205 axis was probably involved in the contribution of resveratrol to AKI in sepsis.

In conclusion, intake of resveratrol was conducive to reliving inflammation and AKI in sepsis by suppressing the MALAT1/miR-205 axis. However, several pitfalls could limit the reliability of this conclusion. Firstly, now we were unable to recruit AKI patients with resveratrol treatment, so the clinical efficacy of resveratrol on AKI in sepsis could not be visually presented. Secondly, though regulation of MALAT1 on miR-205 was disclosed, how this modulation was formed was not investigated. Thirdly, MALAT1 downstream pathways, which were alterable by resveratrol, were not studied in depth. The points raised above all demand solid proof in future.

\section{Acknowledgements}

This work was supported by the Scientific Research Projects of Hunan Health Commission (No: 20201105), the Scientific and Technological Development Project of Chenzhou Municipal Science and Technology Bureau (No: zdyf201926 and No: ZDYF2020052) and the Natural Science Project of Xiangnan University (No: 2019XJ70).

The authors declare no conflict of interest.

\section{References}

1. Jia P, Teng J, Zou J, et al. (2015): Xenon protects against septic acute kidney injury via miR-21 target signaling pathway. Crit Care Med 43: e250-259.

2. Sheng B, Zhao L, Zang X, et al. (2017): miR-375 ameliorates sepsis by downregulating miR-21 level via inhibiting JAK2STAT3 signaling. Biomed Pharmacother 86: 254-261.

3. Wang H, Bei Y, Shen S, et al. (2016): miR-21-3p controls sepsis-associated cardiac dysfunction via regulating SORBS2. J Mol Cell Cardiol 94: 43-53.

4. Li Z, Deng X, Kang Z, et al. (2016): Elevation of miR-21, through targeting MKK3, may be involved in ischemia pretreatment protection from ischemia-reperfusion induced kidney injury. J Nephrol 29: 27-36.

5. Li C, Wu J, Li Y, Xing G (2017): Cytoprotective effect of heat shock protein 27 against lipopolysaccharide-induced apoptosis of renal epithelial HK-2 cells. Cell Physiol Biochem 41:2211-2220.

6. Uchino S, Kellum JA, Bellomo R, et al. (2005): Acute renal failure in critically ill patients: a multinational, multicenter study. JAMA 294: 813-818.

7. Fu D, Dong J, Li P, et al. (2017): MiRNA-21 has effects to protect kidney injury induced by sepsis. Biomed Pharmacother 94: 1138-1144.

8. Jia P, Wu X, Dai Y, et al. (2017): MicroRNA-21 is required for local and remote ischemic preconditioning in multiple organ protection against sepsis. Crit Care Med 45: e703-e710.

9. Rauf A, Imran M, Butt MS, et al. (2018): Resveratrol as an anti-cancer agent: A review. Crit Rev Food Sci Nutr 58: 1428-1447.

10. Bonnefont-Rousselot D (2016): Resveratrol and cardiovascular diseases. Nutrients 8: 250.

11. Malaguarnera L (2019): Influence of resveratrol on the immune response. Nutrients 11: 946.

12. Wang Y, Feng F, Liu M, et al. (2018): Resveratrol ameliorates sepsis-induced acute kidney injury in a pediatric rat model via Nrf2 signaling pathway. Exp Ther Med 16: 3233-3240.

13. Wang N, Mao L, Yang L, et al. (2017): Resveratrol protects against early polymicrobial sepsis-induced acute kidney injury through inhibiting endoplasmic reticulum stress-activated NF-kappaB pathway. Oncotarget 8: 36449-36461.

14. Boshra SA (2020): Resveratrol modulates miR-34a in cardiotoxicity induced by isoproterenol. J Med Food 23: 593-599.

15. Wen W, Chen X, Huang Z, et al. (2020): Resveratrol regulates muscle fiber type conversion via miR-22-3p and AMPK/ SIRT1/PGC-1alpha pathway. J Nutr Biochem 77: 108297.

16. Ge YT, Zhong AQ, Xu GF, Lu Y (2019): Resveratrol protects BV2 mouse microglial cells against LPS-induced inflammatory injury by altering the miR-146a-5p/TRAF6/NF-kappaB axis. Immunopharmacol Immunotoxicol 41: 549-557.

17. Wang JF, Yu ML, Yu G, et al. (2010): Serum miR-146a and miR-223 as potential new biomarkers for sepsis. Biochem Biophys Res Commun 394: 184-188.

18. Ji ZR, Xue WL, Zhang L (2019): Schisandrin B attenuates inflammation in LPS-induced sepsis through miR-17-5p downregulating TLR4. Inflammation 42: 731-739. 
19. Tili E, Michaille JJ, Alder H, et al. (2010): Resveratrol modulates the levels of microRNAs targeting genes encoding tumor-suppressors and effectors of TGFbeta signaling pathway in SW480 cells. Biochem Pharmacol 80: 2057-2065.

20. Wang W, Lou C, Gao J, et al. (2018): LncRNA SNHG16 reverses the effects of miR-15a/16 on LPS-induced inflammatory pathway. Biomed Pharmacother 106: 1661-1667.

21. Gutschner T, Hammerle M, Diederichs S (2013): MALAT1 - a paradigm for long noncoding RNA function in cancer. J Mol Med (Berl) 91: 791-801.

22. Chen H, Wang X, Yan X, et al. (2018): LncRNA MALAT1 regulates sepsis-induced cardiac inflammation and dysfunction via interaction with miR-125b and p38 MAPK/NFkappaB. Int Immunopharmacol 55: 69-76.

23. Geng F, Liu W, Yu L (2019): Potential role of circulating long noncoding RNA MALAT1 in predicting disease risk, severity, and patients' survival in sepsis. J Clin Lab Anal 33: e22968.

24. Li J, Wang J, Chen Y, et al. (2016): LncRNA MALAT1 exerts oncogenic functions in lung adenocarcinoma by targeting miR-204. Am J Cancer Res 6: 1099-1107.

25. Chen Y, Qiu J, Chen B, et al. (2018): Long non-coding RNA NEAT1 plays an important role in sepsis-induced acute kidney injury by targeting miR-204 and modulating the NF-kappaB pathway. Int Immunopharmacol 59: 252-260.

26. Chen Q, Huang X, Li R (2018): IncRNA MALAT1/miR-205$5 \mathrm{p}$ axis regulates MPP(+)-induced cell apoptosis in MN9D cells by directly targeting LRRK2. Am J Transl Res 10: 563572.

27. Lin Y, Ding Y, Song S, et al. (2019): Expression patterns and prognostic value of miR-210, miR-494, and miR-205 in middle-aged and old patients with sepsis-induced acute kidney injury. Bosn J Basic Med Sci 19: 249-256.

28. Doi K, Leelahavanichkul A, Yuen PS, Star RA (2009): Animal models of sepsis and sepsis-induced kidney injury. J Clin Invest 119: 2868-2878.

29. Song S, Meyer M, Turk TR, et al. (2009): Serum cystatin C in mouse models: a reliable and precise marker for renal function and superior to serum creatinine. Nephrol Dial Transplant 24: 1157-1161.

30. Sun IO, Shin SH, Cho AY, et al. (2017): Clinical significance of NGAL and KIM-1 for acute kidney injury in patients with scrub typhus. PLoS One 12: e0175890.

31. Haddy FJ (2004): Acute renal failure and sepsis. N Engl J Med 351: 2347-2349; author reply 2347-2349.

32. Yohannes S, Chawla LS (2009): Evolving practices in the management of acute kidney injury in the ICU (Intensive Care Unit). Clin Nephrol 71: 602-607.

33. Akcay A, Nguyen Q, Edelstein CL (2009): Mediators of inflammation in acute kidney injury. Mediators Inflamm 2009: 137072 .

34. Kim MY, Lim JH, Youn HH, et al. (2013): Resveratrol prevents renal lipotoxicity and inhibits mesangial cell glucotoxicity in a manner dependent on the AMPK-SIRT1-PGC1alpha axis in db/db mice. Diabetologia 56: 204-217.

35. Qi B, Shi C, Meng J, et al. (2018): Resveratrol alleviates ethanol-induced neuroinflammation in vivo and in vitro: Involvement of TLR2-MyD88-NF-kappaB pathway. Int J Biochem Cell Biol 103: 56-64.

36. Byun EB, Sung NY, Park JN, et al. (2015): Gamma-irradiated resveratrol negatively regulates LPS-induced MAPK and NF-kappaB signaling through TLR4 in macrophages. Int Immunopharmacol 25: 249-259.
37. Pellegrina D, Severino P, Barbeiro HV, et al. (2017): Insights into the function of long noncoding RNAs in sepsis revealed by gene co-expression network analysis. Noncoding RNA 3: 5 .

38. Dai Y, Liang Z, Li Y, et al. (2017): Circulating long noncoding RNAs as potential biomarkers of sepsis: a preliminary study. Genet Test Mol Biomarkers 21: 649-657.

39. Li Z, Chao TC, Chang KY, et al. (2014): The long noncoding RNA THRIL regulates TNFalpha expression through its interaction with hnRNPL. Proc Natl Acad Sci U S A 111: 1002-1007.

40. Cui H, Xie N, Tan Z, et al. (2014): The human long noncoding RNA lnc-IL7R regulates the inflammatory response. Eur J Immunol 44: 2085-2095.

41. Zhao G, Su Z, Song D, et al. (2016): The long noncoding RNA MALAT1 regulates the lipopolysaccharide-induced inflammatory response through its interaction with NF-kappaB. FEBS Lett 590: 2884-2895.

42. Jia P, Wu N, Jia D, Sun Y (2019): Downregulation of MALAT1 alleviates saturated fatty acid-induced myocardial inflammatory injury via the miR-26a/HMGB1/TLR4/NF-kappaB axis. Diabetes Metab Syndr Obes 12: 655-665.

43. Zhang Z, Cai CX (2016): Kidney injury molecule-1 (KIM-1) mediates renal epithelial cell repair via ERK MAPK signaling pathway. Mol Cell Biochem 416: 109-116.

44. Chen L, Feng P, Zhu X, et al. (2016): Long non-coding RNA Malat1 promotes neurite outgrowth through activation of ERK/MAPK signalling pathway in N2a cells. J Cell Mol Med 20: 2102-2110.

45. Huang W, Lan X, Li X, et al. (2017): Long non-coding RNA PVT1 promote LPS-induced septic acute kidney injury by regulating TNFalpha and JNK/NF-kappaB pathways in HK-2 cells. Int Immunopharmacol 47: 134-140. 